\title{
Enhanced Transmission and Receiver Diversity in Orthogonal Frequency Division Multiplex Systems Using Blind Channel Estimation
}

\author{
Vivek Kumar Gupta ${ }^{1}$, Sandip Vijay \\ ${ }^{1}$ Uttarakhand Technical University, India \\ ${ }^{2}$ ICFAI UNIVERSITY, India
}

\begin{tabular}{l} 
Article Info \\
\hline Article history: \\
Received Oct 25, 2017 \\
Revised Jan 4, 2018 \\
Accepted Aug 12, 2018 \\
\hline
\end{tabular}

Keyword:

BER

Blind channel estimation

OFDM

Receiver diversity

\begin{abstract}
This paper deals with the blind channel estimation technique in OFDM system with receiver diversity to analyze the bit error rate with respect to the number of symbols. The paper clearly brings out the advantage that is being offered by the use of Blind channel estimation technique in terms of SNR requirements. Also a comparative study has been made for the analysis of BER variation with the amount i.e. number of symbols being transmitted. The work also explores the possibility of obtaining an optimum value of number of receivers that may lead to desired BER for threshold value of SNR in an OFDM system.
\end{abstract} All rights reserved.

\section{Corresponding Author:}

Vivek Kumar Gupta,

Uttarakhand Technical University, Dehradun, Uttarakhand, India.

Email:vivekgupta79ster@gmail.com

\section{INTRODUCTION}

Channel estimation forms an important part of communication systems. It is used for detecting the received signal, improving efficacy of orthogonal frequency division multiple access (OFDMA) systems and improving the BER of the system. Channel estimation can be classified into three categories namely Training-based channel estimation, Blind channel estimation and Semi blind channel estimation. The blind channel estimation utilizes the statistical information of the channel and certain characteristics of the transmitted signals. Although improved bandwidth efficiency can be obtained in blind techniques due to the lack of training overhead, the convergence speed and estimation accuracy are comparatively poor. Additionally, the time-varying nature of the channel in wireless applications forces the pilot/training sequence to be transmitted periodically, resulting in degraded channel throughput.

Channel estimation plays an important role in digital communication systems. For estimating a channel, its transfer function has to be identified directly or indirectly. The problem originates from the fact that the transmitted signal to an unknown channel is not available at the receiver input. Hence the conventional MMSE approaches are not applicable. The possible resolution to the blind identification problem can be found in [1] which utilizes the higher order statistics of the channel output. On the contrary [2] presented results that are based on the second order statistic. The basic blind channel estimation process utilizes a channel model shown in Figure 1, where only the observation signal is available for processing in the identification and estimation of channel. Training sequences tends to use more transmission bandwidth, for a time varying channel. Hence blind estimation techniques exploiting a known property of the source have become popular [3]. These usually exploit the second or higher order statistics [4] for channel estimation. The statistical methods rely on assumptions on the input sequence statistics while the latter 
method make no such assumption [5], [6]. Deterministic methods are generally suggested when the input statistics are unknown, or there is insufficient time samples for estimation. Blind estimation methods are blind in the sense that the system (channel) input is not used in the estimation but they do require some statistical assumptions. In the scenario of not enough data samples to find a reasonably accurate statistical estimate, it may be assumed that the estimates of the signal are close enough to their true (known) values. Hence, it may be easier to solve this problem by treating the input as a deterministic signal which has been done in [5],[6].

\section{PROPOSED RESEARCH METHOD}

Orthogonal frequency-division multiplexing (OFDM) combined with multiple antennas have become significant in its capability to overcome the multipath fading and boosting the system capacity, [7]. The SS method having simple structure achieves good performance, but it leads to several difficulties when applied to MIMO OFDM systems [8],[9]. Firstly, more number of receive antennas than transmit antennas are essential which adversely effects the symmetry of links in wireless transmission standards, e.g. the $2 \times 2$ MIMO for IEEE 802.11n equipment. However, for oversampling at the receiver, the case $A_{R} \leq A_{T}$ is also possible [10]. Besides, equal number of the transceiver antennas is obviously used in the current SISO OFDM transmission schemes, e.g. IEEE 802.11a standard. Secondly, for the systems with more receive antennas, the knowledge of the channel order must be obtained, which is not very convenient. Considering $A_{T}$ transmit and $A_{R}$ receive antennas for a MIMO-OFDM system, employing $A_{C}$ subcarriers. Let $x[k] \stackrel{\text { def }}{=}$ $\left[x 1[k] x 2[k] \ldots \ldots \ldots A_{T}[k]^{T}\right]$ and $y[k] \stackrel{\text { def }}{=}\left[y 1[k] y 2[k] \ldots \ldots \ldots y A_{R}[k]^{T}\right]$. Where $x_{q}[k]$ and $y_{p}[k]$ the transmitted symbols at the qth are transmit antenna and the symbol received at the pth receive antenna respectively. Hence the input vector can be written as.

$$
\left.x \stackrel{\text { def }}{=} x[0]^{T} x[1]^{T} \ldots \ldots x\left[A_{C}-1\right]^{T}\right]^{T}
$$

and the observation

$$
\left.y \stackrel{\text { def }}{=} y[0]^{T} y[1]^{T} \ldots \ldots y[A c-1]^{T}\right]^{T}
$$

Let the noise be $\mathrm{n}$, then the input-output relation of the MIMO-OFDM system may be expressed by

$$
\mathrm{y}=\mathrm{Hx}+\mathrm{n}
$$

Where matrix $\mathrm{H} \stackrel{\text { def }}{=} \operatorname{diag}\left(\mathrm{H}[0] \cdot\right.$ - $\left.\quad \mathrm{H}\left[A_{C}-1\right]\right)$ has size $\left(A_{R} A_{C}\right) \times\left(A_{T} A_{C}\right)$, with diagonal blocks defined as

$$
\mathrm{H}[\mathrm{K}]=\left[\begin{array}{cccc}
h_{1,1}[k] & h_{1,2}[k] & \ldots & h_{1, A_{T}}[k] \\
h_{2,1}[k] & h_{2,2}[k] & \ldots & h_{1, A_{T}}[k] \\
\vdots & \vdots & \ddots & \vdots \\
h_{A_{R}, 1}[k] & h_{A_{R}, 2}[k] & \ldots & h_{A_{R}, A_{T}}[k]
\end{array}\right]
$$

Where $h_{p, q}[k]$ denotes the frequency response from the qth transmit to the pth receive antenna over the kth subcarrier. In this paper point of significance is the blind estimation of the channel coefficients, i.e., $h_{p, q}[k]$, directly from the observation y.

The proposed approach aims at analyzing the variation of bit error rates for OFDM systems using blind channel estimation. OFDM systems utilizing the blind channel estimation have been used numerous times as they offer advantages of optimum bandwidth with maximum data rates which in combination with OFDM also leads to reduced inter symbol interference. The signal is BPSK modulated by modulating the orthogonal subcarriers and is then transmitted over the channel. The system used in this approach utilizes the blind channel estimation technique with subspace based approach. It utilizes the concept of receiver diversity which is tested for a system with five receiving antennas.

Using the subspace based approach for blind channel estimation, the received signal is estimated \& compared for BER with the transmitted signal. The process starts with analyzing the BER with a receiver diversity scenario which is initialized using a single receiver and is further continued by increasing the number of receivers up to a maximum of five. A gradual trend is observed in the values obtained for SNR required for a desired value of BER i.e. bit error rate. This trend is analyzed for different number of receiver antennas and finally a conclusion can be obtained from the values of SNR requirement and the desired BER. 
Another aspect of the proposed work can be explored by observing the behavior of SNR requirement in accordance with the increase in the number of symbols transmitted which directly analyses the efficiency of the system with the increase in its transmission capacity. this for and also a detailed analysis is made by varying the number of receivers for a single value of $\mathrm{N}$ i.e. the number of symbols being transmitted .Also the analyses is continued by varying the amount of transmitted data i.e. the number of symbols and observing the trend with respect to the amount of data transmitted.

\section{RESULTS AND ANALYSIS}

As can be inferred from the Figure 1 with the receiver diversity ranging from 1 to 5 analysis of bit error rate for the system gives an interesting relationship with the signal to noise ratio (SNR) requirement for a desired value of BER. It is observed that with gradual increase in the number of receivers although there is an increase in the SNR requirement for which an optimum number of receivers can be easily obtained through the observations of the curves. This analysis was further continued so as to find an amicable solution for the SNR requirements for a desirable and acceptable error probability for high data rates or for higher values of N. It can be deduced from the curves of BER Vs SNR for various values of N. The values of SNR requirement for desirable BER for values of $\mathrm{N}=104,105$ \& 106 analyzed and observed to be following a trend which clearly indicates that irrespective of the number of symbols being transmitted i.e. the amount of the data transmitted, the SNR requirement of the OFDM system with blind channel estimation technique for a desired value of BER i.e. bit error rate does not show any major shift in the values in terms of Signal to noise ratio, hence for the modern communication systems which generally work at high data rates whether it may be $3 \mathrm{G}$ or $4 \mathrm{G} / \mathrm{LTE}$ this conclusion can serve as a milestone for deciding the receivers specifications and parameters. Some of the interpretations from Figure 1-Figure 4 can be summarized as under:

a. With increasing values of SNR for different data rates or ' $N$ ', BER the values more closely fall in line with the theoretical results i.e. the difference between the theoretical and simulated values decreasesFor lower values of desired bit error rates the SNR requirements increase irrespective of the diversity scenario or the number of receiving antennas.

b. For achieving a desired bit error rates the system with less number of receivers shows better performance with reduced SNR power requirement.

c. It can be observed from Figure 1, Figure 2, Figure 3 \& Figure 4 that for different values of $\mathrm{N}$ i.e. at $102,103,105$ \& 106 the SNR requirement for a desired value of BER i.e. bit error rate in a receiver diversified scenario does not change considerably hence this observation indicates that quality of a signal can be monitored by keeping a check on the signal SNR level in a receiver diversity system which can be further utilized for improved data rates for OFDM systems.

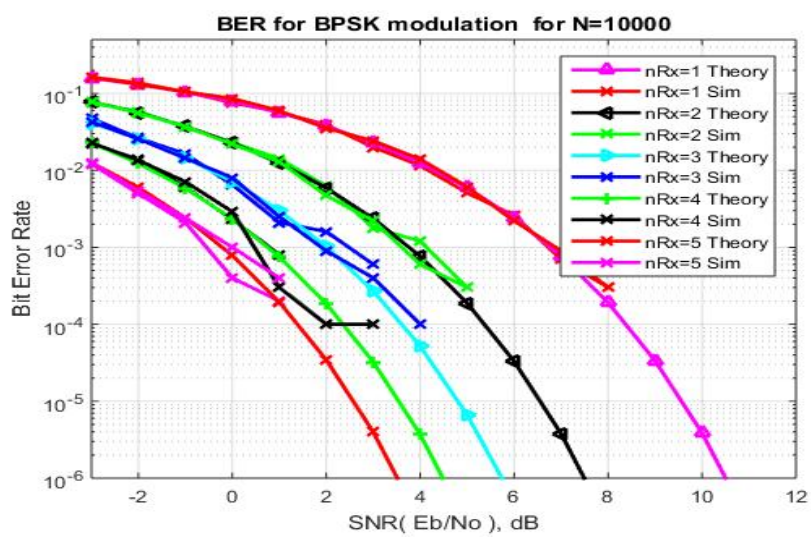

Figure 1. BER for BPSK for $\mathrm{N}=103$

As can be inferred from the curves of Figure 1, Figure 2, Figure 3 and Figure 4 that with only a slight change in the Signal to Noise power ratio at the receiver there can be tremendous improvement in the amount of data that can be transmitted in communication system that too also with a desired range of Bit Error. 


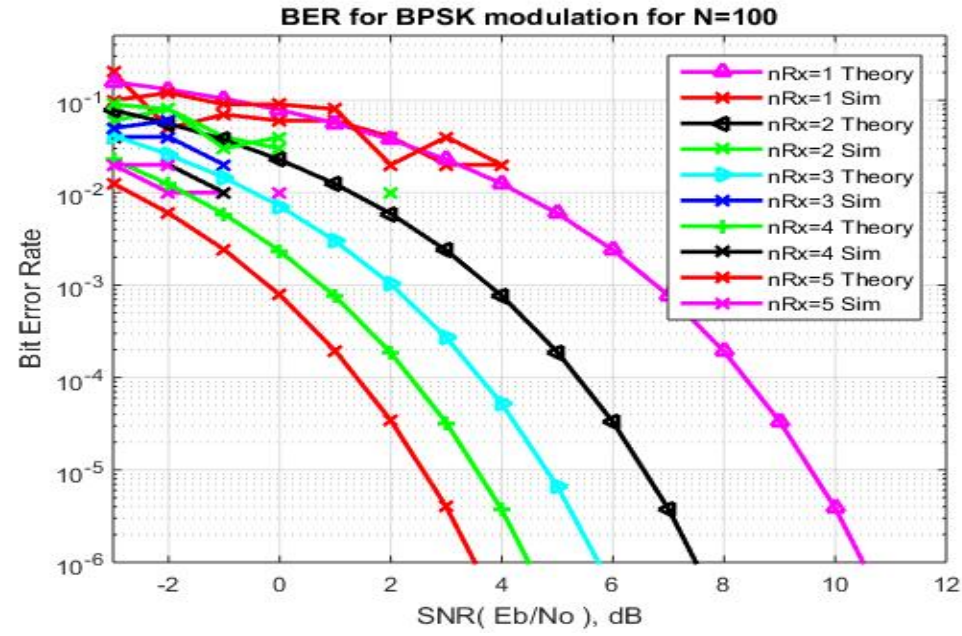

Figure 2. BER for BPSK for $\mathrm{N}=102$

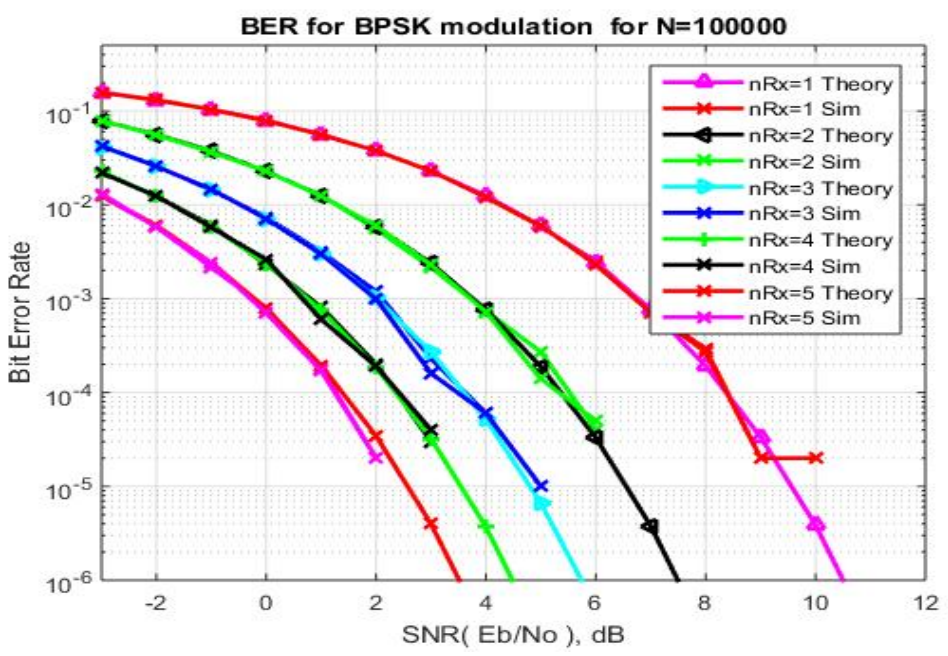

Figure 3. BER for BPSK for $\mathrm{N}=105$

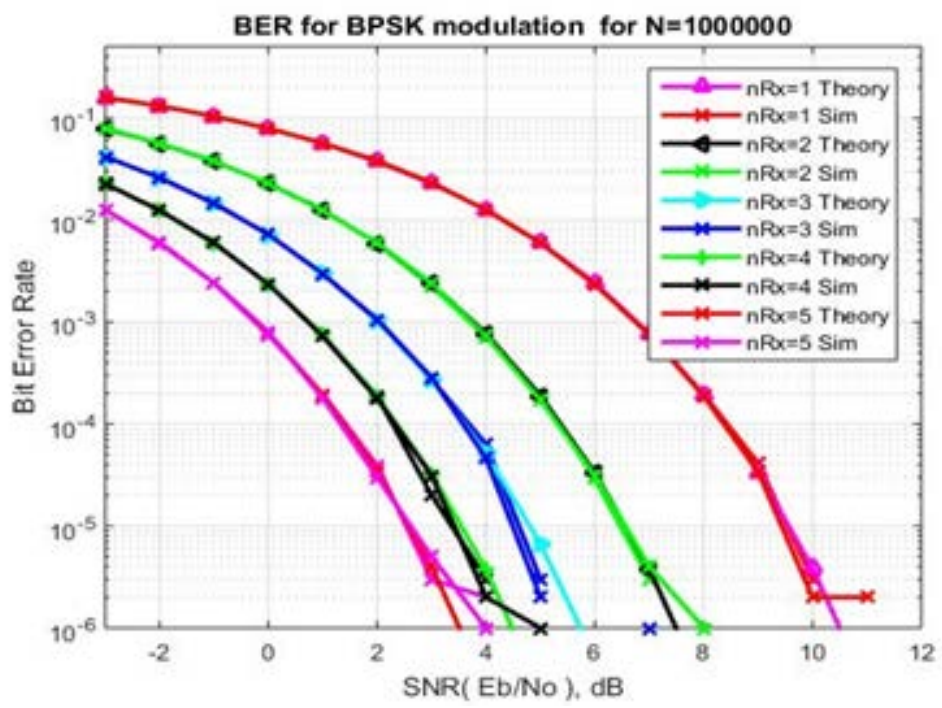

Figure 4. BER for BPSK for $\mathrm{N}=106$ 
Rate the given curves of Figure 1, Figure 2, and Figure 3 and Figure 4 justify the above conclusion with reference to the variation in the values of $\mathrm{N}$, i.e. the number of symbols transmitted which is also an indicator of the transmitted data.

\section{CONCLUSION}

The proposed work in this paper aimed at the analysis of an OFDM system in context to the Signal to noise power ratio required at the receiver for correct estimation of the received data. The work firstly observed the SNR requirement with respect to a desired BER in a receiver diversity scenario in which it was found that there is a significant amount of shift in power for received signal for different number of receivers but this can be easily optimized by selecting the appropriate number of receivers. Another aspect explored in this work was that irrespective of the number of symbols being transmitted i.e. the amount of the data transmitted, the SNR requirement of the OFDM system with blind channel estimation technique for a desired value of BER i.e. bit error rate does not show any major shift in the values in terms of Signal to noise ratio, hence for the modern communication systems which generally work at high data rates whether it may be 3G or $4 \mathrm{G} / \mathrm{LTE}$ this conclusion can serve as a milestone for deciding the receivers specifications and parameters.

\section{REFERENCES:}

[1]. R. A. Kennedy and Z. Ding, "Blind Adaptive Equalizers for QAM Communication Systems Based on Convex Cost Functions", SPIE Optical Engineering. June 1992.

[2]. L.Tong, G.Xu and T.Kailath. "A new approach to blind identification and equalization of multipath channel.," Proc. Aailomar Conf. Signal, Systems and computers, November 1991.

[3]. J. A. Cadzow, "Blind deconvolution via cumulant extrema:' IEEE Signal Processing Magazine, vol. 13, no. 3, pp. 2442.

[4]. May 1996.

[5]. L. Tong, G. Xu, and T. Kailath. "Blind identification and equalization based on second-order statistics: A time domain approach,'” IEEE Transactions on Information Theory, vol. 40, no. 2, pp. 340-349, Mar. 1994.

[6]. G. Xu, H. Liu, L. Tong, and T. Kailath, "A least-squares approach to blind channel identification,” IEEE Trans. Signal Process., vol. 43, no. 12, pp. 2982-2993, Dec. 1995.

[7]. G. B. Giannakis and C. Tepedelenlioglu, "Direct blind equalizers of multiple FIR channels: A deterministic approach,” IEEE Trans. Signal Process., vol. 47, no. 1, pp. 62-74, Jan. 1999.

[8]. G. J. Foschini, and M. J. Gans, "On limits of wireless communications in a fading environment when using multiple antennas,” Wireless Personal Commun., vol. 6, pp. 311-335, 1998.

[9]. E. de Carvalho, and D. Slock, "Blind and semi-blind FIR multi-channel estimation: (global) identifiability conditions,” IEEE Trans. [9]Signal Processing, vol. 52, pp. 1053-1064, Apr. 2004.

[10]. K. Abed-Meraim, P. Loubaton, and E. Moulines, "A subspace algorithm for certain blind identification problem," IEEE Trans. Inform. Theory, vol. 32, pp. 499-511, Apr. 1997.

[11]. C. Shin, R. W. Heath, Jr., and E. J. Powers, "Blind channel estimation for MIMO-OFDM systems,” IEEE Trans. Veh. Technol., vol. 56, no. 2, pp. 670-685, Mar. 2007.

[12]. TGn Sync Proposal Technical Specification IEEE 802.11-05/1095r2.

[13]. Y. Zeng, and T. S. Ng, "A semi-blind channel estimation method for multiuser multiantenna OFDM systems," IEEE Trans. Signal Processing, vol. 52, pp. 1419-1429, May, 2004.

[14]. E. Moulines, P. Duhamel, J. F. Cardoso, and S. Mayrargue, "Subspace methods for the blind identification of multichannel FIR filters,” IEEE Trans. Signal Processing, vol. 43, pp. 516-525, Feb. 1995.

[15]. Y. Li and Z. Ding, "Blind channel identification based on second order cyclostationary statistics," in Proc. IEEE Int. Conf. Acoust., Speech, Signal Process., Apr. 1993, vol. 4, pp. 81-84.

[16]. F. Gao, Y. Zeng, A. Nallanathan, and T.-S. Ng, "Robust subspace blind channel estimation for cyclic prefixed MIMO OFDM systems: Algorithm, identifiability and performance analysis,” IEEE J. Sel. Areas Commun., vol. 26, no. 2, pp. 378-388, Feb. 2008

[17]. L. Tong, G. Xu, and T. Kailath, "Blind identification and equalization based on second-order statistics: A time domain approach,” IEEE Trans. Inf. Theory, vol. 40, no. 2, pp. 340-349, Mar. 1994.

[18]. L. Tong and S. Perreau, "Multichannel blind identification: From subspace to maximum likelihood methods," Proc. IEEE, vol. 86, no. 10, pp. 1951-1968, Oct. 1998.

[19]. L. Tong, G. Xu, B. Hassibi, and T. Kailath, "Blind channel identification based on second-order statistics: A frequency-domain approach,” IEEE Trans. Inf. Theory, vol. 41, no. 1, pp. 329-334, Jan. 1995.

[20]. Surinder Singh,Sandeep Singh Gill," Performance Evaluation of Channel Estimation in OFDM System for Different QAM and PSK Modulations”, International Journal of Electrical and Computer Engineering (IJECE),Vol 1 No 2, 2011 pages 140-150

[21]. Anis Charrada, Tunisia,” Support Vector Machines Regression for MIMOOFDM Channel Estimation”, IAES International Journal of Artificial Intelligence (IJ-AI),Vol 1 No 4, 2012 pages 214-224 


\section{BIOGRAPHIES OF AUTHORS}

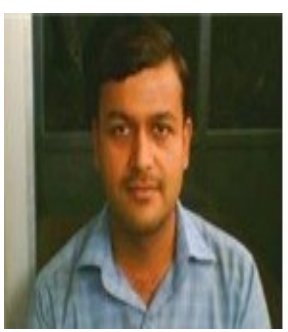

Mr.Vivek Kumar Gupta,Research received B.Tech Degree from CCSU university,after that he received his M.Tech degree from Uttarakhand technical university,Dehradun,Uttarakhand

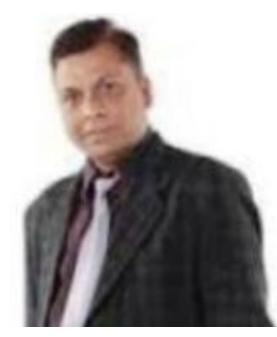

Dr.Sandip Vijay is an eminent Professor of ICFAI University and has guided many scholars in Ph.d,also he has publication in many international journals of repute 